\title{
Reactive Intermediates
}

Volume 2 


\title{
Reactive Intermediates
}

\section{Volume 2}

\author{
Edited by \\ R.A.Abramovitch
}

Clemson University

Clemson, South Carolina 
Library of Congress Cataloging in Publication Data

Main entry under title:

Reactive intermediates, 2

Includes bibliographies and index.

1. Chemical reaction, Conditions, and laws of-Addresses, essays, lectures. I. Abramovitch, R. A., 1930-

QD501.R345

$541^{\prime} 39$

79-344

ISBN-13: 978-1-4613-3194-0 e-ISBN-13: 978-1-4613-3192-6

DOI: 10.1007/ 978-1-4613-3192-6

(C) 1982 Plenum Press, New York

Softcover reprint of the hardcover 1st edition 1982

A Division of Plenum Publishing Corporation

233 Spring Street, New York, N.Y. 10013

All rights reserved

No part of this book may be reproduced, stored in a retrieval system, or transmitted, in any form or by any means, electronic, mechanical, photocopying, microfilming, recording, or otherwise, without written permission from the Publisher 


\section{Contributors}

André Baretta, Université d'Aix-Marseille, Centre de Saint-Jérôme, Laboratoire de Stéréochimie, rue H. Poincaré, 13397 Marseille Cédex 4, France.

Per H.J. Carlsen, Department of Chemistry, Emory University, Atlanta, Georgia 30322.

Albert Padwa, Department of Chemistry, Emory University, Atlanta, Georgia 30322.

Manfred G. Reinecke, Department of Chemistry, Texas Christian University, Fort Worth, Texas 76129.

E.F.V. Scriven, Department of Chemistry and Applied Chemistry, University of Salford, Salford M5 4WT, United Kingdom. Present address: Reilly Tar and Chemical Corporation, 1510 Market Square Center, 151 N. Delaware Street, Indianapolis, Indiana 46204.

J-M. Surzur, U.D.E.S.A.M., Faculté des Sciences et Technique (St. Jérôme), 13397 Marseille Cédex 13, France.

Yi-Noo Tang, Department of Chemistry, Texas A\&M University, College Station, Texas 77840.

Bernard Waegell, Université d'Aix-Marseille, Centre de Saint-Jérôme, Laboratoire de Stéréochimie, rue H. Poincaré, 13397 Marseille Cédex 4, France. 


\section{Preface}

The field of reactive intermediates has been blossoming at a rapid rate in recent years and its impact on chemistry, both "pure" and "applied," as well as on biology, astronomy, and other areas of science, is enormous. Several books have been published which cover the area; one, edited by McManus,* surveys the subject in general at the senior undergraduate or beginning graduate level. In addition, a number of monographs have appeared which deal with individual topics such as carbenes, nitrenes, free radicals, carbanions, carbenium ions, and so on, in great depth.

Our objective is somewhat different. We hope that these Advances in ... type of volumes will appear at irregular intervals of a year to 18 months each. We intend to publish up-to-date reviews in relatively new areas of the chemistry of reactive intermediates. These will be written by world authorities in the field, each one of whom will give the reader a current in-depth review of all aspects of the chemistry of each of these species. It is our plan that the subjects to be reviewed will cover not only organic chemistry but also inorganic, physical, bio-, industrial, and atmospheric chemistry. The volumes themselves, we hope, will end up being reasonably interdisciplinary, though this need not and probably will not be the case for the individual reviews. It is our intention to give readers ideas about the importance of reactive intermediates in fields other than their own, as well as to bring them up to date in their own individual areas.

I welcome suggestions of topics (and authors) that should be discussed in future volumes.

Clemson, S.C.

R. A. Abramovitch

* Samuel P. McManus, Ed., Organic Reactive Intermediates, Vol. 26 in Organic Chemistry, Academic Press, New York (1973). 


\section{Contents}

\section{Current Aspects of the Solution Chemistry of Arylnitrenes}

E. F. V. Scriven

I. Introduction . . . . . . . . . . . . . . . . . . . . . . 1

1. Definition and Historical . . . . . . . . . . . . . . . . . . . 1

2. Nomenclature . . . . . . . . . . . . . . . . . . . . . . . . 2

3. Methods of Generation and Evidence for Arylnitrenes . . . . . . . . . . 2

A. Thermolysis of Aryl Azides . . . . . . . . . . . . . . . . . . . 2

B. Photolysis of Aryl Azides . . . . . . . . . . . . . . . . . . . 4

C. Reduction of Nitro and Nitrosoarenes . . . . . . . . . . . . . . . 4

D. Fragmentation of Heterocycles . . . . . . . . . . . . . . . . . 5

E. Oxidation of Amines . . . . . . . . . . . . . . . . . . . . . 7

F. Newer Methods of Generation . . . . . . . . . . . . . . . . . . 8

4. Spectral, Structural, and Theoretical Considerations . . . . . . . . . . . 8

II. Intermolecular Reactions . . . . . . . . . . . . . . . . . . . . . . 11

1. Singlet Reactions . . . . . . . . . . . . . . . . . . . . . . . . 12

A. Aromatic Substitution . . . . . . . . . . . . . . . . . . . . . 12

B. Addition to Alkenes . . . . . . . . . . . . . . . . . . . . . . . 14

C. Reactions with Nitriles . . . . . . . . . . . . . . . . . . . . 15

D. Trapping by Carbon Monoxide . . . . . . . . . . . . . . . . . 16

E. Electrophilic Substitution at Nitrogen . . . . . . . . . . . . . . . 16

2. Triplet Reactions . . . . . . . . . . . . . . . . . . . . . . . 18

A. Aniline Formation . . . . . . . . . . . . . . . . . . . . . . 18

B. "Dimerization" . . . . . . . . . . . . . . . . . . . . . . . 18

C. Insertion into Aliphatic C-H Bonds . . . . . . . . . . . . . . . . 21

D. Reaction with Nitrosoarenes . . . . . . . . . . . . . . . . . . 22

E. Reaction with Oxygen . . . . . . . . . . . . . . . . . . . . 23

F. Substitution of a Carbon-Fluorine Bond . . . . . . . . . . . . . . 24

3. "Benzazirine" Reactions . . . . . . . . . . . . . . . . . . . . . 25

A. With Nucleophiles . . . . . . . . . . . . . . . . . . . . . . 25

B. With Electrophiles . . . . . . . . . . . . . . . . . . . . . . 34

III. Intramolecular Reactions . . . . . . . . . . . . . . . . . . . . . . 36

1. Nitrene-Induced Cyclizations . . . . . . . . . . . . . . . . . . . 36

A. To form Five-Membered Rings . . . . . . . . . . . . . . . . . 36

B. Closure to Six-Membered Rings . . . . . . . . . . . . . . . . . . . 39

2. Heterocyclic Rearrangements . . . . . . . . . . . . . . . . 44 
IV. Decomposition in the Presence of Protonic and Lewis Acids . . . . . . . . . 45

V. Current and Future Application . . . . . . . . . . . . . . . . . . . . 47

Appendix . . . . . . . . . . . . . . . . . . . . . . . . . . . . . . 48

References . . . . . . . . . . . . . . . . . . . 49

2. Nitrile Ylides and Nitrenes From $2 H$-Azirines

Albert Padwa and Per H. J. Carlsen

I. Generation of Nitrile Ylides . . . . . . . . . . . . . . . . . . . . . 56

II. Features of the Photocycloaddition Reaction of $2 \mathrm{H}$-Azirines . . . . . . . . 58

III. Photochemical Dimerizations of $2 H$-Azirines . . . . . . . . . . . . . . . 68

IV. Intramolecular 1,5-Electrocyclization Reactions of Vinyl Substituted $2 H$-Azirines $\quad 71$

V. Properties of Nitrile Ylides . . . . . . . . . . . . . . . . . . . . . 78

VI. Intramolecular Photocycloaddition Reactions of $2 \mathrm{H}$-Azirines . . . . . . . . 80

VII. Further Reactions of $2 \mathrm{H}$-Azirines . . . . . . . . . . . . . . . . . . 91

VIII. Thermal Reactions of $2 \mathrm{H}$-Azirines . . . . . . . . . . . . . . . . . . 96

References . . . . . . . . . . . . . . . . . . . . 113

\section{Radical Cyclizations by Intramolecular Additions}

\section{$J-M$. Surzur}

I. Introduction . . . . . . . . . . . . . . . . . . . . . . . . . 121

II. General Presentation: The Early Results . . . . . . . . . . . . . . . . 123

1. The Unstabilized 5-Hexenyl Radicals . . . . . . . . . . . . . . . . . . . 123

2. The Stabilized 5-Hexenyl Radicals . . . . . . . . . . . . . . . . . 126

III. Attempts to Rationalize the Selectivities Observed in the Cyclization of

5-Hexenyl Radicals . . . . . . . . . . . . . . . . . . . . . . . . 126

1. The Irreversible Pathway: Why Is the $(\mathrm{Cy} \cdot 5)$ Radical Favored? . . . . . . 127

A. The Steric Hypothesis . . . . . . . . . . . . . . . . . . . . 127

B. The Stereoelectronic Hypothesis . . . . . . . . . . . . . . . . 128

C. The Entropic Hypothesis . . . . . . . . . . . . . . . . . . . 130

D. Conclusion . . . . . . . . . . . . . . . . . . . . . . . . 131

2. The Reversible Pathway Favoring the $\left(\mathrm{Cy}^{\circ} 6\right)$ Radical . . . . . . . . . . 131

IV. Alkyl Substituted 5-Hexenyl Radicals . . . . . . . . . . . . . . . . . 133

V. Alkenyl Radicals Other than 5-Hexenyl . . . . . . . . . . . . . . . . 137

1. The Higher Homologs . . . . . . . . . . . . . . . . . . . . . 137

2. The Lower Homologs . . . . . . . . . . . . . . . . . . . . . . 139

A. The 4-Pentenyl Radicals . . . . . . . . . . . . . . . . . . . 139

B. The 3-Butenyl Radicals . . . . . . . . . . . . . . . . . . . . 141

C. The Allyl Radical . . . . . . . . . . . . . . . . . . . . . . 145

3. Conclusion . . . . . . . . . . . . . . . . . . . . . . . . . . 145

VI. Carbon-Centered Alkenyl Radicals Containing Heteroatoms . . . . . . . . 145

1. Introduction . . . . . . . . . . . . . . . . . . . . . . . . . 145

2. Unsaturated Radicals Bearing a Heteroatom in the Chain . . . . . . . . 146

3. Diallylic Substrates . . . . . . . . . . . . . . . . . . . . . . . 148

4. Unsaturated Radicals Bearing Fluorine Atoms on the Chain . . . . . . . 150

VII. Alkenyl Radicals Bearing Stabilizing Groups on the Carbon Radical Center . . 151

1. Introduction . . . . . . . . . . . . . . . . . . . . . . . 151

2. The 5-Hexenyl- and 5-Hexenylalkyl-Substituted Radicals . . . . . . . . 152

3. Other Alkenyl Radicals . . . . . . . . . . . . . . . . . . . . . 154 
4. The Stabilizing Groups . . . . . . . . . . . . . . . . . . . . . 154

5. Conclusion . . . . . . . . . . . . . . . . . . . . . . . . . . 159

VIII. Heteroatom-Centered Radicals . . . . . . . . . . . . . . . . . . . 159

1. Introduction . . . . . . . . . . . . . . . . . . . . . . . 159

2. Oxygen-Centered Radicals . . . . . . . . . . . . . . . . . . . . 160

A. Alkoxyl Radicals . . . . . . . . . . . . . . . . . . . . . . 160

B. Aryloxyl Radicals . . . . . . . . . . . . . . . . . . . . . . 163

C. Acyloxyl Radicals . . . . . . . . . . . . . . . . . . . . . 164

D. Peroxyl Radicals . . . . . . . . . . . . . . . . . . . . . . 167

3. Nitrogen-Centered Radicals . . . . . . . . . . . . . . . . . . . 168

A. Aminyl Radicals : . . . . . . . . . . . . . . . . . . . . . 168

B. Arylaminyl Radicals . . . . . . . . . . . . . . . . . . . . . 174

C. Amidyl Radicals . . . . . . . . . . . . . . . . . . . . . . 175

D. Nitroxide Radicals . . . . . . . . . . . . . . . . . . . . . 176

4. Sulfur-Centered Radicals . . . . . . . . . . . . . . . . . . . . 177

A. Unsaturated Thiyl Radicals Generated from Unsaturated Mercaptans . . 177

B. Unsaturated Thiyl Radicals Generated from Unsaturated Sulfides or Disulfides . . . . . . . . . . . . . . . . . . . . . 185

C. Enethiyl-Unsaturated Radicals, Arylthiyl-Unsaturated Radicals and the Thio-Claisen Rearrangement . . . . . . . . . . . . . . . . . . 187

5. Other Heteroatom-Centered Radicals . . . . . . . . . . . . . . . . 189

A. Phosphorus-Centered Radicals . . . . . . . . . . . . . . . . . 189

B. Silicon-Centered Radicals . . . . . . . . . . . . . . . . . . . 190

C. Germanium-Centered Radicals . . . . . . . . . . . . . . . . . 191

D. Tin-Centered Radicals . . . . . . . . . . . . . . . . . . . . 191

E. Selenium-Centered Radicals . . . . . . . . . . . . . . . . . . 192

6. Conclusion . . . . . . . . . . . . . . . . . . . . . . . . . 192

IX. Intramolecular Addition to Other Carbon-Carbon Bonds . . . . . . . . . . . 193

1. Introduction . . . . . . . . . . . . . . . . . . . . . . . . . 193

2. Intramolecular Addition to Acetylenic Bonds of Carbon-Centered Radicals . 193

3. Intramolecular Addition of Heteroatomic Radicals to Acetylenic Bonds . . . 196

4. Intramolecular Addition to Allenes and Cyclopropanes . . . . . . . . . 199

X. Intramolecular Addition to Polar Multiple Bonds Such as Carbonyl or Cyano $\quad 200$

1. Intramolecular Addition to the Carbonyl Bond . . . . . . . . . . . . 200

A. The $\beta$-Scission Process . . . . . . . . . . . . . . . . . . . . 200

B. The Intramolecular Addition . . . . . . . . . . . . . . . . . . 201

C. Conclusion . . . . . . . . . . . . . . . . . . . . . . . . 207

2. Intramolecular Addition to Carboxylic Acids and Esters . . . . . . . . 207

3. Intramolecular Addition to the Cyano Group . . . . . . . . . . . . . 209

4. Intramolecular Addition to Other Polar Bonds . . . . . . . . . . . . 211

A. The $\mathrm{C}=\mathrm{S}$ Bond . . . . . . . . . . . . . . . . . . . . . . . 211

B. The $\mathrm{C}=\mathrm{N}-$ Bond . . . . . . . . . . . . . . . . . . . . . . 212

C. The $-\mathrm{N}=\mathrm{N}-$ Bond . . . . . . . . . . . . . . . . . . . . . . 214

D. The Nitroso and Nitrone Groups . . . . . . . . . . . . . . . . 214

E. The Nitro Group . . . . . . . . . . . . . . . . . . . . . 215

F. The Azido Group . . . . . . . . . . . . . . . . . . . . . . 215

G. The Sulfone Group . . . . . . . . . . . . . . . . . . . . . 216

5. Conclusion . . . . . . . . . . . . . . . . . . . . . . . . . . 216

XI. Synthesis of Bi- and Polycyclic Compounds. Stereochemical Features of Free

Radical Intramolecular Additions . . . . . . . . . . . . . . . . . . 216

1. Introduction . . . . . . . . . . . . . . . . . . . . . . 216 
2. Bicyclic Compounds . . . . . . . . . . . . . . . . . . . . . . 217

A. The Cy5/Cy6 Case . . . . . . . . . . . . . . . . . . . . . 217

B. The $\mathrm{Cy} 6 / \mathrm{Cy} 7$ Case . . . . . . . . . . . . . . . . . . . . . 232

C. The Cy4/Cy5 Case . . . . . . . . . . . . . . . . . . . . . 235

D. The $\mathrm{Cy} 3 / \mathrm{Cy} 4$ Case . . . . . . . . . . . . . . . . . . . . . 236

E. The Allyl Radical . . . . . . . . . . . . . . . . . . . . . . 239

3. Stereochemical Features of Free Radical Intramolecular Addition . . . . . 239

4. Polycyclization Reactions . . . . . . . . . . . . . . . . . . . . 242

A. Carbon-Centered Radicals . . . . . . . . . . . . . . . . . . 242

B. Heteroatom-Centered Radicals . . . . . . . . . . . . . . . . . 250

C. Polycyclization Reactions Involving Addition to a Polar Bond . . . . . 252

5. Conclusion . . . . . . . . . . . . . . . . . . . . . . . . . . 253

XII. Free Radical Intramolecular Addition as a Mechanistic Tool and a Kinetic

Standard . . . . . . . . . . . . . . . . . . . . . . 253

1. The Mechanistic Tool . . . . . . . . . . . . . . . . . . . 254

A. The 5-Hexenyl Radical . . . . . . . . . . . . . . . . . . . . 254

B. Other Carbon-Centered Radicals . . . . . . . . . . . . . . . . 257

C. Heteroatomic Radicals . . . . . . . . . . . . . . . . . . . . 260

D. Reactions Related to Free Radical Intramolecular Additions _ . . . . . 261

E. Comments on the Use of Unsaturated Systems Cyclization as a Free
Radical Proof . . . . . . . . . . . . . . . . . 269 2606

2. The Kinetic Standard . . . . . . . . . . . . . . . . . . 270

XIII. Conclusion . . . . . . . . . . . . . . . . . . . . . . . . . . . 276

References .. . . . . . . . . . . . . . . . . . 277

4. Reactions of Silicon Atoms and Silylenes Yi-Noo Tang

I. Introduction . . . . . . . . . . . . . . . . . . . . . . . . . . . 297

II. Methods of Generation of Silicon Atoms . . . . . . . . . . . . . . . . 299

1. Nuclear Recoil Method . . . . . . . . . . . . . . . . . . . . . . 299

2. Thermal Evaporation Method . . . . . . . . . . . . . . . . . . 299

III. Reaction Modes of Silicon Atoms . . . . . . . . . . . . . . . . . . . 300

1. Si-H Bond Insertion . . . . . . . . . . . . . . . . . . . . . . . 300

2. Apparent H Abstraction . . . . . . . . . . . . . . . . . . . . . 300

3. Apparent F Abstraction . . . . . . . . . . . . . . . . . . . . . 301

4. Possible Si-Si Bond Insertion . . . . . . . . . . . . . . . . . . . 302

5. Apparent 1,4 Addition to Dienes . . . . . . . . . . . . . . . . . . 302

6. Reactions of Silicon with $\mathrm{SiX}_{4}$ and Other Halides . . . . . . . . . . . . 303

7. Reactions of Si Species from $(n, \gamma)$ Reactions . . . . . . . . . . . . . 303

IV. Modes of Formation of Silylenes . . . . . . . . . . . . . . . . . . . 305

1. Derived from Si Atoms and Elemental Silicon . . . . . . . . . . . . . 305

A. Si Atom Abstraction-the Nuclear Recoil Method . . . . . . . . . . 305

B. Si Atom Insertion-the Co-Condensation Method . . . . . . . . . . 305

C. Disproportionation Involving Silicon-the High-Temperature Reaction Method . . . . . . . . . . . . . . . . . . . 306

D. Abstraction from Single-Crystal Silicon-the Molecular Beam Method . . 306

2. The Decomposition Method . . . . . . . . . . . . . . . . . . . 307

A. From Monosilanes . . . . . . . . . . . . . . . . . . . . . . 307

B. From Disilanes . . . . . . . . . . . . . . . . . . . . . . . 310 
C. From Trisilanes . . . . . . . . . . . . . . . . . . . . . . . 316

D. From Polysilanes . . . . . . . . . . . . . . . . . . . . . . . 318

E. From Silacyclopropanes . . . . . . . . . . . . . . . . . . . 318

F. From 7-Silanorbornadienes . . . . . . . . . . . . . . . . . . . 319

G. From Metal Silyls . . . . . . . . . . . . . . . . . . . . . . 319

3. The Reduction Method . . . . . . . . . . . . . . . . . . . . . . 320

A. Reduction with $\mathrm{H}_{2}$. . . . . . . . . . . . . . . . . . . . . . . 320

B. Reduction with Metallic Elements . . . . . . . . . . . . . . . . 320

V. Spectroscopic and Thermodynamic Properties of Silylenes . . . . . . . . . . 321

1. Electronic States . . . . . . . . . . . . . . . . . . . . . . . . 321

A. Ground Electronic States of Silylenes . . . . . . . . . . . . . . . 321

B. Electronic States of Reacting Silylenes . . . . . . . . . . . . . . . 322

2. Structure . . . . . . . . . . . . . . . . . . . . . . . . 323

3. Heats of Formation . . . . . . . . . . . . . . . . . . . . . . . 323

VI. Chemical Properties of Silylenes . . . . . . . . . . . . . . . . . . . 323

1. Polymerization Reactions . . . . . . . . . . . . . . . . . . . . . 326

A. Dimerization . . . . . . . . . . . . . . . . . . . . . . . . 326

B. Polymerization . . . . . . . . . . . . . . . . . . . . . . . . 335

2. Insertion Reactions . . . . . . . . . . . . . . . . . . . . . . . 335

A. Si-H bonds . . . . . . . . . . . . . . . . . . . . . . . . . . . . . . . . . . . . . . . . . 337

B. Si-Si bonds . . . . . . . . . . . . . . . . . . . . . . . . . 340

C. Si-O bonds . . . . . . . . . . . . . . . . . . . . . . . . . 341

D. Halogen-Containing Bonds . . . . . . . . . . . . . . . . . . . 342

E. Other Hydrogen-Containing Bonds . . . . . . . . . . . . . . . . 342

3. Addition Reactions . . . . . . . . . . . . . . . . . . . . . . . 343

A. Olefins . . . . . . . . . . . . . . . . . . . . . . . . . . . 343

B. Conjugated Dienes . . . . . . . . . . . . . . . . . . . . 352

C. Alkynes . . . . . . . . . . . . . . . . . . . . . . . . . . 356

D. Aromatic Compounds . . . . . . . . . . . . . . . . . . . . . 359

E. Ketones . . . . . . . . . . . . . . . . . . . . . . . . . . 360

References . . . . . . . . . . . . . . . . . . . 360

\section{Five-Membered Hetarynes} Manfred G. Reinecke

I. Introduction . . . . . . . . . . . . . . . . . . . . . . . . . . . 367

1. Role and Scope . . . . . . . . . . . . . . . . . . . . . . . . . 367

2. Formulas and Nomenclature . . . . . . . . . . . . . . . . . . . . 368

A. Carbocyclic Arynes . . . . . . . . . . . . . . . . . . . . . . 368

B. Hetarynes . . . . . . . . . . . . . . . . . . . . . . . . . 370

C. Related Heterocyclic Species . . . . . . . . . . . . . . . . . . 371

3. Historical Perspective . . . . . . . . . . . . . . . . . . . . . . 373

A. Development of the Aryne Concept . . . . . . . . . . . . . . . . 373

B. History of the Five-Membered Hetaryne Hypothesis . . . . . . . . . 375

II. Chemistry of Arynes . . . . . . . . . . . . . . . . . . . . . . . . 377

1. Methods of Preparation . . . . . . . . . . . . . . . . . . . . . . 377

A. General Considerations . . . . . . . . . . . . . . . . . . . . 377

B. Arynes from Aryl Anion Intermediates _. . . . . . . . . . . . . . . 378

C. Arynes by Loss of Nitrogen from Acyclic Substituents . . . . . . . . 379 
D. Arynes by Loss of Nitrogen from Cyclic Systems _ . . . . . . . . . . 384

E. Arynes from Other Cyclic Systems . . . . . . . . . . . . . . . . 389

F. Arynes from Other Sources . . . . . . . . . . . . . . . . . . . 396

2. Methods of Detection . . . . . . . . . . . . . . . . . . . . . . 398

A. Cine-Substitution . . . . . . . . . . . . . . . . . . . . . . . . . . . . . . 399

B. Cycloaddition . . . . . . . . . . . . . . . . . . . . . . . . 404

III. Five-Membered Hetarynes . . . . . . . . . . . . . . . . . . . . . . 414

1. Oxaarynes . . . . . . . . . . . . . . . . . . . . . . . . . . . 414

A. 2,3-Didehydrobenzofuran . . . . . . . . . . . . . . . . . . . . 414

B. 2,3-Didehydrofuran . . . . . . . . . . . . . . . . . . . . . . 418

C. Didehydromaleic Anhydride . . . . . . . . . . . . . . . . . . 419

2. Azaarynes . . . . . . . . . . . . . . . . . . . . . . . . . . . 423

A. 2,3-Didehydroindole . . . . . . . . . . . . . . . . . . . . . 423

B. 2,3-Didehydropyrrole . . . . . . . . . . . . . . . . . . . . 427

C. 3,4-Didehydropyrrole . . . . . . . . . . . . . . . . . . . . . 430

D. Didehydromaleimide . . . . . . . . . . . . . . . . . 430

3. Thiaarynes . . . . . . . . . . . . . . . . . . . . . . 432

A. 2,3-Didehydrothiophene . . . . . . . . . . . . . . . . . 432

B. 3,4-Didehydrothiophene . . . . . . . . . . . . . . . . . . . . 466

C. 2,3-Didehydrothianaphthene . . . . . . . . . . . . . . . . . . 471

D. 2,3-Didehycrothianaphthene-1,1-dioxide . . . . . . . . . . . . . . 476

4. Selenaarynes . . . . . . . . . . . . . . . . . . . . . . . . . 478

5. Diazaarynes .. . . . . . . . . . . . . . . . . . . . . . . . . . . . . 479

A. Didehydroimidazole . . . . . . . . . . . . . . . . . . . . . . 479

B. Didehydropyrazoles . . . . . . . . . . . . . . . . . . . . . . 485

6. Thiazaarynes . . . . . . . . . . . . . . . . . . . . . . . . . . 487

A. Didehydrothiazole . . . . . . . . . . . . . . . . . . . . . . 487

B. Didehydroisothiazoles . . . . . . . . . . . . . . . . . . . . . 488

C. Didehydro-1,2,5-thiadiazole . . . . . . . . . . . . . . . . . . . 490

7. Benzdidehydro Five-Membered Heterocycles . . . . . . . . . . . . . . 491

A. Benzdidehydrooxoles . . . . . . . . . . . . . . . . . . . . . 491

B. Benzdidehydroazoles . . . . . . . . . . . . . . . . . . . . . 492

C. Benzdidehydrothioles . . . . . . . . . . . . . . . . . . . . . 495

D. Didehydroazepinoazoles and Thioles . . . . . . . . . . . . . . . 497

8. Five-Membered Carbocyclic Arynes . . . . . . . . . . . . . . . . . 497

A. Didehydroferrocene . . . . . . . . . . . . . . . . . . . . . . 497

B. Didehydrocyclopentadiene Anion . . . . . . . . . . . . . . . . . 500

9. Hetarynium Species . . . . . . . . . . . . . . . . . . . 501

IV. Summary . . . . . . . . . . . . . . . . . . . . . . . . . 505

1. Electronic Factors . . . . . . . . . . . . . . . . . . . . . . 505

2. Geometric Factors . . . . . . . . . . . . . . . . . . . . 506

3. Kinetic Factors . . . . . . . . . . . . . . . . . . . . . . . . . 508

4. Prognosis . . . . . . . . . . . . . . . . . . . . . . . 509

References and Notes . . . . . . . . . . . . . . . . . . . . 510 
6. A Survey of Favorskii Rearrangement Mechanisms. Influence of the Nature and Strain of the Skeleton

André Baretta and Bernard Waegell

I. Introduction . . . . . . . . . . . . . . . . . . . . . . . . . . . 527

1. Favorskii reaction mechanisms . . . . . . . . . . . . . . . 529

II. Symmetrical Mechanisms . . . . . . . . . . . . . . . . . . . . . . 531

1. Cyclopropanone Mechanism and Experimental Evidence for the Symmetrical Intermediate . . . . . . . . . . . . . . . . . . . . . . 531

2. First Step of the Mechanism . . . . . . . . . . . . . . . . . 532

A. Cyclohexane Substrates . . . . . . . . . . . . . . . . . . . 533

3. Second Step of the Mechanism . . . . . . . . . . . . . . . . . . 536

A. Open-Chain Juxtacyclic Substrates . . . . . . . . . . . . . . 536

B. Cyclohexane Substrates . . . . . . . . . . . . . . . . . . . . 539

C. Influence of Structural Features on the Reactivity of Acyclic and Cyclic Substrates ...................... 552

4. Relative Stability of Zwitterion and Cyclopropanone . . . . . . . . . . . 557

A. Acyclic Substrates . . . . . . . . . . . . . . . . . . . . . . 557

B. Cyclic Substrates . . . . . . . . . . . . . . . . . . 560

5. Conclusion . . . . . . . . . . . . . . . . . . . . . . 562

III. Unsymmetrical Mechanism . . . . . . . . . . . . . . . . . 566

1. Occurrence . . . . . . . . . . . . . . . . . . . . . . . . . 566

2. Evidence for the Semibenzilic Mechanism . . . . . . . . . . . . 567

A. Open-Chain Juxtacyclic Substrates . . . . . . . . . . . . . . . . 567

B. Cyclic Substrates . . . . . . . . . . . . . . . . . . . . . . . 568

3. Competition of the Cyclopropanone and the Semibenzilic Mechanisms as a
Function of the Ring Strain and of the Base Strength . . . . . . . . . . 570

4. Conclusion . . . . . . . . . . . . . . . . . . . . . . . . . . 572

IV. The Favorskii Rearrangement in Bridged Polycyclic and Cage Compounds . . . 573

1. Results and Discussion . . . . . . . . . . . . . . . . . . . . . . 573

2. Secondary Reactions in Bridged Bicyclic and Cage Compounds . . . . . . . 577

3. Conclusion . . . . . . . . . . . . . . . . . . . . . . . 580

V. General Conclusion . . . . . . . . . . . . . . . . . . . 580

References . . . . . . . . . . . . . . . . . . . 582

Index ....................... 587 